\title{
Two Novel Mutations in LAMC2 Gene in Iranian Families Affected by Junctional Epidermolysis Bullosa
}

\author{
Maryam Taghdiri ${ }^{1}$, Sirous Naeimi*1, Majid Fardaei², \\ Seyed Mohammad Bagher Tabei ${ }^{2,3}$
}

\begin{abstract}
Background: Junctional epidermolysis bullosa (JEB) is an autosomal recessive skin disorder with defective adhesion of dermal- epidermal within the lamina lucida region of the basement membrane zone. The main characterization of JEB is blistering and fragile skin and mucous membrane. Laminins are noncollagenous part of basement membrane and classified as a family of extracellular matrix glycoprotein. Laminins contain three chains: Laminin $\alpha$, Laminin $\beta$ and Laminin $\gamma$. LAMC2 (laminin subunit gamma 2) gene encodes $\gamma$ subunit of laminin and its mutation contributes to JEB. Here, we report a disease-causing nonsense mutation and a large deletion mutation in LAMC2 gene in two families affected by JEB.

Methods: Whole exome sequencing (WES) was carried out on the mother of patient in family I and the patient himself in family II to detect the underlying mutations. Then, sanger sequencing was performed to confirm the identified mutations.

Results: Next generation sequencing (NGS) data analysis of the first family showed a novel, nonsense mutation in LAMC2 gene (LAMC2: NM_005562: exon14:c.C2143T: p.R715X). The heterozygous state of the mutation was confirmed by sanger sequencing in the parents and unaffected brother. In Family II, NGS data had no coverage in the large area of $L A M C 2$ gene. Thus, to confirm the possible deletion sanger sequencing was done and blasting of sequence showed the deleted region of $9.4 \mathrm{~kb}$ (exon10-17) in LAMC2 gene.

Conclusions: In summary, current study reported a novel disease-causing premature termination codon (PTC) mutation in $L A M C 2$ gene and a large deletion mutation in patients affected by JEB.
\end{abstract}

Keywords: Junctional Epidermolysis Bullosa, LAMC2 gene, Novel mutation, Skin disorder.

\section{Introduction}

Epidermolysis bullosa (EB) is a group of heritable, severe fragile skin disorders in human and animal. It is classified into different subclasses based on the underlying genes and symptoms. Regarding to the severity and type of EB, blisters and skin deformity can appear in distinct part of the whole body $(1,2)$.

Junctional epidermolysis bullosa (JEB) is an autosomal recessive skin disorder with defective adhesion of dermal- epidermal within the lamina lucida of the basement membrane zone. This can be attributed to defective proteins such as laminin 332, collagen XVII and integrin $\alpha 6 \beta 4$ or integrin $\alpha 3$. The main characterization of JEB is blistering and fragile skin and mucous membrane along with little or no trauma. Clinically, JEB is classified into two major 
groups: Herlitz and non-herlitz. In the former type, early onset of severe symptoms is observed and lead to early death, while in the latter one affected individuals demonstrate blisters during their normal lifespan (3).

Laminines are noncollagenous part of basement membrane and classified as a family of extracellular matrix glycoprotein. Laminines contain three chains: Laminin $\alpha$, Laminin $\beta$, and Laminin $\gamma$. Laminin 332 can act as a bridge between the epidermis and the underneath dermis. It is an extracellular adhesion ligand which associated with the hemidesmosomeanchoring filament complex. Mutations of three genes (LAMA3, LAMB3, and LAMC2) encoding the three subunits of laminin 332, affect dermal-epidermal adhesion and mechanically induced skin blistering and fragility, which are the main characteristic of JEB $(4,5)$. The herlitz type mainly shows biallelic premature termination codon (PTC) mutation in one of the laminin 332 gene, while non-herlitz forms have missense mutation in one or both alleles. This is why the herlitz type is more life threatening than non-herlitz type (3).

The LAMC2 (laminin subunit gamma 2) gene with 23 exons is located on q25-q31 location of chromosome 1 and highly expressed in skin and lung tissues $(6,7)$. In this study, we report a disease-causing nonsense mutation and a large deletion in LAMC2 in two families affected by JEB.

\section{Materials and Methods}

The Ethics Committee of the Islamic Azad University of Kazerun approved the study protocol. The patients and all family members signed a written informed consent to participate in this study.

\section{Family I, patient I}

The patient I, was a product of full-term vaginal delivery. She had nail dystrophy, red scars on the face, abdomen, and legs. Loss of serum protein, electrolytes and dermal sepsis seemed to have been responsible for death one month after birth, with extensive blisters and erosions. Unfortunately, the skin biopsy of the patient and blood sample were not accessible. The parents were second- degree cousins and had one healthy child. The mother also reported three previous spontaneous abortions. The patient was passed away at the time of genetic evaluations.

\section{Family II, patient II}

The male infant was born with numerous skin erosion and fragility of mucous membrane. There was extensive absence of skin on the face, trunk, and limbs. There was positive history of EB in his extended families, thus he was referred to genetic counseling to find the underlying mutation for his severe condition. The patient died 1 week after birth due to severe wounds and respiratory failure.

\section{Whole-Exome Sequencing (WES)}

Whole exome sequencing (WES) was carried out on the mother of patient in family I and the patient himself in family II to capture and enrich all exons and crucial flanking site of $L A M C 2$ gene. Next generation sequencing (NGS) was conducted using Illumina Hiseq 2000 machine to sequence about 100 million reads and standard Illumina protocol for pairend 99 nucleotide sequencing. Data was aligned with a reference genome by BWA aligner, variants was identified by GATK and then annotated by ANNOVAR as a functional bioinformatics tool (8-10).

\section{Sanger Sequencing}

Sanger sequencing was performed on parents and healthy child of family I in order to confirm the WES results and also find the possible inheritance manner of the mutations. Patient was expired at the time of experiment thus we do not have access to the sample. Whole blood samples were collected from participants in Ethylenediamine tetraacetic acid (EDTA) tubes. DNA was extracted from blood using QIAamp DNA Minikit (Qiagen, Germany) according to the instructions. DNA concentrations were assessed using NanoDrop C (Thermofisher, USA) and then kept at $-20{ }^{\circ} \mathrm{C}$ until use. Primer pairs are listed in Table 1. 
Table 1. Sequences of primers.

\begin{tabular}{llll}
\hline Gene, Exon number & Primer Sequence & Length (bp) & TM $\left({ }^{\circ} \mathbf{C}\right)$ \\
\hline LAMC2, Exon 14 & $\begin{array}{l}\text { F: TTTGACCATAAGCCAGTCAA } \\
\text { R: TCCCAACACAGCAGTATTC }\end{array}$ & 345 & 54 \\
\hline LAMC2, Exon 9-18 & $\begin{array}{l}\text { F: CAGTGTATATGTCCTGTTGGG } \\
\text { R: CGCTTGAACTCATCCATATG } \\
\text { (Use Primer pairs of exon 14 as inner primers). }\end{array}$ & 10660 & 54 \\
\hline
\end{tabular}

\section{Results}

Next generation sequencing (NGS) data analysis of the first family showed a novel, nonsense (stop gain) mutation in LAMC2 gene (LAMC2: NM_005562: exon14:c.C2143T: p.R715X).

The heterozygous state of the mutation was confirmed by sanger sequencing in the parents and unaffected brother. Therefore, this mutation might be inherited as autosomal recessive manner and mutation in this gene is associated with recessive JEB disease (Fig. 1a). Although we did not access to patient's sample, according to bioinformatics tools such as mutation taster (11) and mutation assessor this substitution variant is disease causing. Moreover, comparative amino acid alignment of $L A M C 2$ protein was performed using $\mathrm{T}$-coffee multiple sequence alignment online program across different animal kingdoms. The result showed that Arg715 residue of the protein was highly conserved through the evolution (Fig. 1b).

In Family II, NGS data had no coverage in the large area of $L A M C 2$ gene. Thus, to confirm the possible deletion primer pairs were designed to identify exact deletion region by sanger sequencing (Fig. 2a). Blasting the sequence data showed the deleted region of $9.4 \mathrm{~kb}$ in $L A M C 2$ gene (Fig. 2b), which encompasses exon 10-17 and so lacking several functional exons would definitely cause severe symptoms in affected individual.

A

B
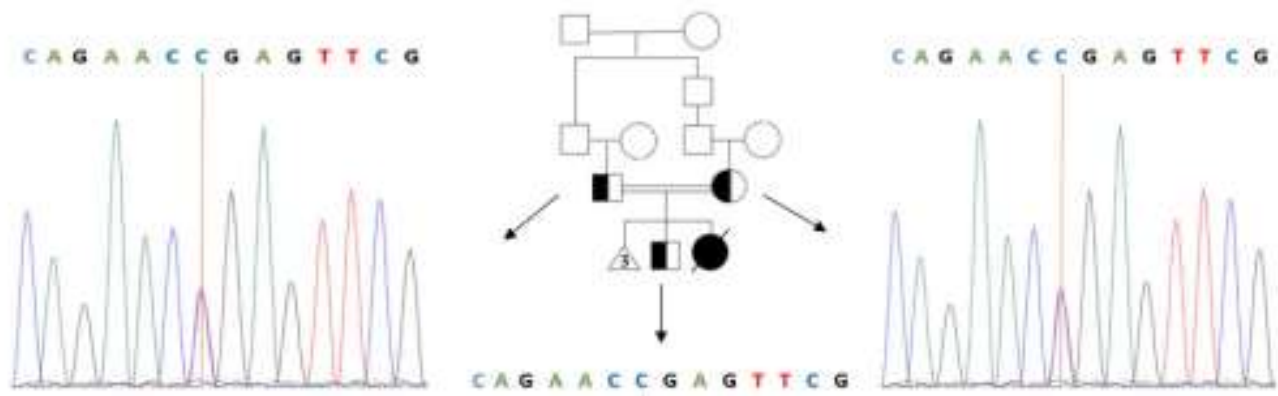

Mouse
Human
Gori1la
Cow
Cat
Panda

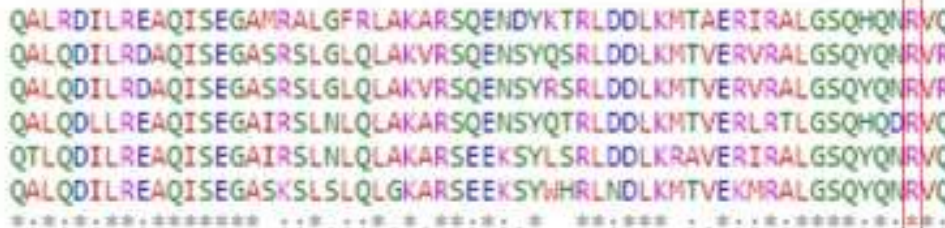

Fig. 1. A) Sanger Sequencing Chromatogram of Family I. B) Comparative multiple sequencing alignment of LAMC2 protein across different animal kingdom. 

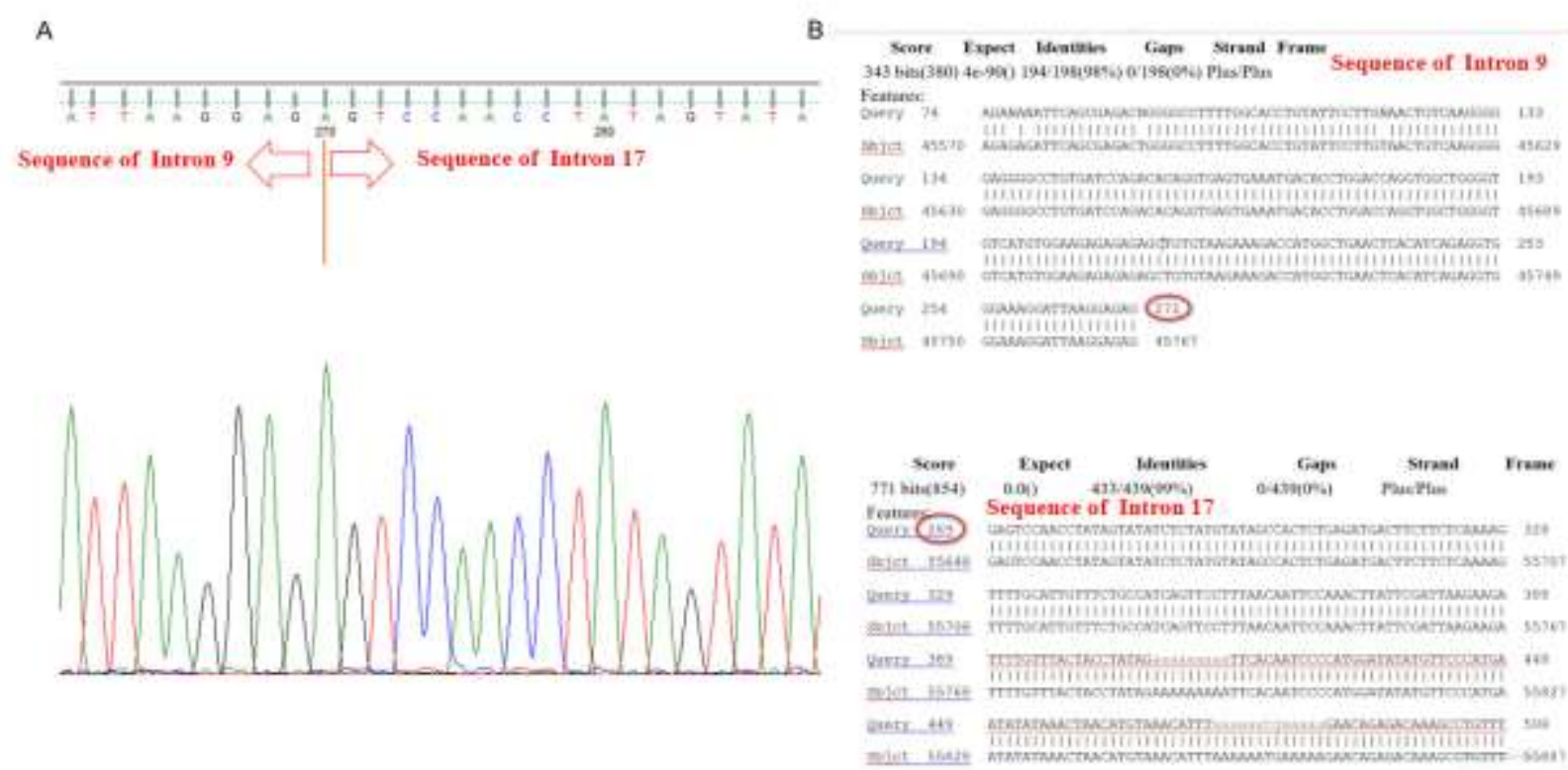

Fig. 2. A) Sanger Sequencing Chromatogram of patient of Family II. B) Blast result of sequence of patient II.

\section{Discussion}

Inherited Junctional epidermolysis bullosa is an autosomal recessive subtype of epidermolysis bullosa disorder, characterized by defective dermal-epidermal adhesion. Herlitz (MIM\# 226700) and non-Herlitz (MIM\# 226650) are two major subgroups of JEB. Herlitz JEB is a severe form which identified with serious blistering over wide regions of the body and mucous membranes of digestive and respiratory tracts, leading to feeding and breathing difficulties, from birth or early ages. The affected individuals usually survive just a few weeks or months (12). The main underlying mutations of JEB occur in the three genes, LAMA3, LAMB3 and $L A M C 2$, encoding the $\alpha 3, \beta 3, \gamma 2$ chains of laminin 332. $L A M C 2$ is located on $1 \mathrm{q} 25.3$, and the total genomic size is approximately $59 \mathrm{~kb}$, according to the UCSC Genome Browser (hg38). In 2002, Nakano et al had investigated JEB mutations in two main forms. In Herlitz form, 13 out of 15 individuals had PTC mutation (3) Jeon et al reported a compound heterozygote mutation ( one missense mutation c.79G> A and an insertion one (382 insT) in LAMC2 gene, which also resulted in PTC mutation (13). Other studies also showed the PTC mutation as a dominant mutation among Herlitz patients in human (14).
In this study we introduce another nonsense mutation which is responsible for causing Herlitz JEB (LAMC2: NM_005562: exon14: c. 2143C> T: p. R715X). This mutation was reported in dbSNP (rs1035597347) but not reported in ClinVar, Human Genome Variation Database and Human Genome Mutation Database. The global minor allele frequency of this SNP is 0.000004 for T allele. According to mutation taster, this mutation can lead to nonsense mediated decay (NMD) process. In the normal protein the stop codon amino acid is at 1194 while in mutant one is located at position 715 . Therefore, protein truncation is obvious.

In the second family, the large deletion was confirmed in affected person and the mutation evaluation showed the deletion of exons 10-17. For further deletion evaluation, the blast result of patient's sanger sequences reveals that the first segment of this sequence (up to the nucleotide 270 ) is located in the intron between 9 and 10 exons, while the rest of the sequence is located in the intron between 17 and 18 exons. Therefore, we came to the conclusion that 10-17 exons were omitted in the homozygous status (Fig 2). This is the first large deletion mutation of LAMC2 gene in human. 
In conclusion, current study reported a novel disease causing PTC mutation in LAMC2 gene and a large deletion mutation in patients affected by JEB-Herlitz type. This finding expands the mutation spectrum of LAMC2 which will be useful for prenatal molecular studies. Further practical studies are needed to evaluate the

\section{References}

1. Boeira VL, Souza ES, Rocha Bde O, Oliveira PD, Oliveira Mde F, Rego VR, et al. Inherited epidermolysis bullosa: clinical and therapeutic aspects. An Bras Dermatol. 2013;88(2):185-98.

2. Fine JD, Bruckner-Tuderman L, Eady RA, Bauer EA, Bauer JW, Has C, et al. Inherited epidermolysis bullosa: updated recommendations on diagnosis and classification. J Am Acad Dermatol. 2014;70(6):1103-26.

3. Nakano A, Chao SC, Pulkkinen L, Murrell D, Bruckner-Tuderman L, Pfendner E, et al. Laminin 5 mutations in junctional epidermolysis bullosa: molecular basis of Herlitz vs. nonHerlitz phenotypes. Hum Genet. 2002;110(1):41-51.

4. Kiritsi D, Has C, Bruckner-Tuderman L. Laminin 332 in junctional epidermolysis bullosa. Cell Adh Migr. 2013;7(1):135-141.

5. Benati D, Miselli F, Cocchiarella F, Patrizi C, Carretero M, Baldassarri S, et al. CRISPR/Cas9Mediated In Situ Correction of LAMB3 Gene in Keratinocytes Derived from a Junctional Epidermolysis Bullosa Patient. Mol Ther. 2018;26(11):2592-2603.

6. Kallunki P, Sainio K, Eddy R, Byers M, Kallunki T, Sariola H, et al. A truncated laminin chain homologous to the B2 chain: structure, spatial expression, and chromosomal assignment. J Cell Biol. 1992;119(3):679-93.

7. Airenne T, Haakana H, Sainio K, Kallunki T, Kallunki P, Sariola H, et al. Structure of the human laminin gamma 2 chain gene (LAMC2): alternative splicing with different tissue distribution of two transcripts. Genomics. 1996;32(1):54-64. effects of this mutation on mRNA and protein expression.

\section{Acknowledgements}

The authors would like to thank the family members for participating in current study.

8. Li H, Durbin R. Fast and accurate short read alignment with Burrows-Wheeler transform. Bioinformatics. 2009;25(14):1754-60.

9. McKenna A, Hanna M, Banks E, Sivachenko A, Cibulskis K, Kernytsky A, et al. The Genome Analysis Toolkit: a MapReduce framework for analyzing next-generation DNA sequencing data. Genome Res. 2010;20(9):1297-303.

10. Wang K, Li M, Hakonarson H. ANNOVAR: functional annotation of genetic variants from high-throughput sequencing data. Nucleic Acids Res. 2010;38(16):e164.

11. Schwarz JM, Cooper DN, Schuelke M, Seelow D. MutationTaster2: mutation prediction for the deep-sequencing age. Nat Methods. 2014;11(4):361-2.

12. Yao YY, Zhang Y, Xie XH, Chen L, Zhu F, Zhou M. Identification of a Novel COL17A1 Compound Heterozygous Mutation in a Chinese Girl with Non-Herlitz Junctional Epidermolysis Bullosa. Curr Med Sci. 2020;40(4):795-800.

13. Jeon IK, Kim SE, Kim SC. Novel compound heterozygous mutation in LAMC2 genes (c.79G $>\mathrm{A}$ and 382insT) in Herlitz junctional epidermolysis bullosa. J Dermatol. 2014;41(4):322-4.

14. Vailly J, Pulkkinen L, Christiano AM, Tryggvason K, Uitto J, Ortonne JP, et al. Identification of a homozygous exon-skipping mutation in the $L A M C 2$ gene in a patient with Herlitz's junctional epidermolysis bullosa. J Invest Dermatol. 1995;104(3):434-7. 